\title{
A Pair Formation Approach to Modeling Inheritance of Social Traits
}

\author{
S. Lubkin* and C. Castillo-Chavez**
}

\begin{abstract}
Transmission of cultural traits behaves superficially like genetic transmission, but is substantially more complicated, since transmission is influenced by the population at large, as is disease transmission. We present a framework for modeling cultural transmission by a system of ordinary differential equations, with nonlinearities both in the transmission and in the formation of pairs. The framework is illustrated with a simple example.
\end{abstract}

\section{Introduction}

Mathematical models of social dynamics have been studied extensively both in the context of demography, and in their own right. There is an extensive literature on marriage functions that goes back to 1949 [9]. Currently, there are two approaches that dominate the modeling of social dynamics. Classical dynamics follow the birth and death processes within the female population [8]. The classical formalism has proven extremely useful in the study of age-dependent mortality and fertility in demographic processes. An alternative approach that is gaining considerable attention consists of using models that follow the dynamics of pairs. This formalism naturally incorporates the processes of pair formation and dissolution in addition to the usual birth and death processes. Pair formation models have been used to study demographic processes, population genetics, and epidemiological processes. For a thorough list of references, see [3].

The availability of an axiomatic framework [3] opens up the possibilities for systematic study of a variety of questions in demography, epidemiology, population genetics, ecology, and social and cultural dynamics. Our objective here is to illustrate the use of our axiomatic approach for the construction of dynamic models that may prove useful in the study of the propagation or survival of social traits such as religion and language. The traditional approach $[2,4]$ has been to track generations, assuming cultural transmission occurs once, and all births are

* Dept. of Mathematics and Statistics, Thackeray Hall, University of Pittsburgh, Pittsburgh, PA 15260

** Biometrics Unit and Center for Applied Mathematics, 337 Warren Hall, Cornell University, Ithaca, NY 14853-7801 
simultaneous. All parameters are probabilities, rather than rates. We will take a mixed approach, incorporating both rates and probabilities.

Section 2 introduces the pair-formation framework, and in Section 3 we derive the general formula for the cultural transmission system. In Section 4, we analyze the simplest nontrivial example of pair formation and cultural transmission.

\section{Classical and modern approaches}

In 1972, Parlett asked, "Can there be a marriage function?" [11]. In mathematical terms, this was asking whether or not it is possible to have a satisfactory mathematical description of heterosexual pair formation, i.e. mathematically valid and biologically relevant. The first answer known to us is provided by the classical demographic pair-formation model $[5,6,9]$. The approach is based on the use of a nonlinear function $\psi$ to model the process (rate) of pair formation. For the situation with one class each of females $f(t)$ and males $m(t)$, the mixing/pair formation function $\psi$ for this heterosexually-active mixing population is assumed to satisfy the following properties at time $t[6,10]$ :

(a) $\psi(0, f)=\psi(m, 0)=0$, i.e. in the absence of either males or females there is no pair formation,

(b) $\psi(\alpha m, \alpha f)=\alpha \psi(m, f) \quad \forall \alpha, m, f \geq 0$, i.e. if the sex ratio remains constant, the increase in pair formation is proportional only to population size, $f$.

(c) $\psi(m+u, f+v) \geq \psi(m, f) \quad \forall \quad u, v, f, m \geq 0$, i.e. $\psi$ is monotonic in $m$ and

Condition (b) implies that most reasonable mixing functions are of the form

$$
\psi(m, f)=m g\left(\frac{f}{m}\right)=f h\left(\frac{m}{f}\right)
$$

In demography and epidemiology, researchers have employed a variety of pair formation functions, including

$$
\begin{gathered}
\psi(m, f)=c \min (m, f) \\
\psi(m, f)=c \sqrt{m f} \\
\psi(m, f)=2 c \frac{m f}{(m+f)}
\end{gathered}
$$

where $c$, an arbitrary positive constant, denotes the rate of pair formation. Pair formation can represent the occurrence of marriages, sexual liaisons, social contacts, etc. 


\section{Formulation of the general model}

\section{Definitions}

Consider a two-sex population divided into $N$ groups. The groups may represent different languages, religions, socio-economic groups, geographic characteristics, etc. For simplicity, assume the number of male groups is the same as the number of female groups, a 50:50 sex ratio in each group, including equal birth rates for males and females, uniform mortality rates, and heterosexual pairing. These assumptions can be relaxed, but doing so does not contribute much to the current discussion, although it may contribute in specific cases where data indicates so. Let

$m_{i}=$ number of single males in group $i$

$f_{i}=$ number of single females in group $i$

$Q_{i j}=$ number of pairs with male of type $i$ and female of type $j$

$M_{i}=\sum_{j} Q_{i j}=$ number of paired $i$ males

$F_{i}=\sum_{j} Q_{j i}=$ number of paired $i$ females

$X_{i}^{l k}=$ proportion of offspring of an $l k$ pair which are of type $i$

$r_{l k}=$ reproductive rate of $l k$ pair

$\Lambda_{i}=\sum_{l} \sum_{k} r_{l k} X_{i}^{l k} Q_{l k}=$ recruitment rate of single males or females of type $i$

$\sigma_{l k}=$ separation rate of $l k$ pair

$\mu=$ mortality rate

$b_{i}=$ per capita pair formation rate of $i$ males

$c_{i}=$ per capita pair formation rate of $i$ females

$p_{i j}=$ proportion of type- $i$ male pairings which are with $j$ females

$q_{i j}=$ proportion of type- $i$ female pairings which are with $j$ males

The above definitions give:

$$
\begin{gathered}
\sum_{j} p_{i j}=\sum_{j} q_{i j}=1 \\
\sum_{i} c_{i} m_{i}=\sum_{i} b_{i} f_{i}
\end{gathered}
$$

\section{Mixing framework}

Following Castillo-Chavez and Busenberg [3], we use an axiomatic framework to describe the probabilities associated with pair formation which form a substantial portion of the basis of our models for the transmission of cultural traits. Specifically, the set of mixing probabilities, $p_{i j}$ and $q_{i j}$, establishes the mixing/pair for- 
mation among individuals of a heterosexually-active population if they satisfy the following postulates:

(A1) $0 \leq p_{i j} \leq 1, \quad 0 \leq q_{i j} \leq 1$

(A2) $\sum_{j} p_{i j}=\sum_{j} q_{i j}=1$

(A3) $c_{i} m_{i} p_{i j}=b_{j} f_{j} q_{j i}$

(A4) If, for some $i$ or $j, c_{i} b_{j} m_{i} f_{j}=0$, then we define $p_{i j}=q_{i j}=0$.

Property (A3) simply expresses conservation of pairings. A useful class of pair formation functions is given by proportionate mixing, or Ross solutions [3],

$$
\begin{gathered}
p_{i j}=\bar{p}_{j}=\frac{b_{j} f_{j}}{\sum c_{i} m_{i}} \\
q_{i j}=\bar{q}_{j}=\frac{c_{j} m_{j}}{\sum b_{i} f_{i}}
\end{gathered}
$$

where, of course, $\sum_{i} c_{i} m_{i}=\sum_{i} b_{i} f_{i}$. It can be shown [3], that all solutions to (A1)-(A4) can be given by multiplicative perturbations of proportionate mixing,

$$
\begin{array}{r}
p_{i j}=\bar{p}_{j}\left(\phi_{i j}+\frac{R_{i}^{m} R_{j}^{f}}{\sum_{k} \bar{p}_{k} R_{k}^{f}}\right) \\
- \\
q_{i j}=\bar{q}_{j}\left(\phi_{i j}+\frac{R_{i}^{f} R_{j}^{m}}{\sum_{k} \bar{q}_{k} R_{k}^{m}}\right)
\end{array}
$$

where $\phi_{i j}$ are preference coefficients which yield deviations from pure proportionate mixing, and $R_{i}^{m}$ is given by $1-\sum_{k} \bar{p}_{k} \phi_{i k}$ and $R_{i}^{f}$ is given by $1-\sum_{k} \bar{q}_{k} \phi_{i k}$.

\section{General model}

With the above mixing framework, we can now write the general model

$$
\begin{aligned}
\dot{m}_{i} & =\Lambda_{i}-\left(\mu+c_{i}\right) m_{i}+\sum_{j}\left(\mu+\sigma_{i j}\right) Q_{i j} \\
\dot{f}_{i} & =\Lambda_{i}-\left(\mu+b_{i}\right) f_{i}+\sum_{j}\left(\mu+\sigma_{j i}\right) Q_{j i} \\
\dot{Q}_{i j} & =c_{i} m_{i} p_{i j}-\left(2 \mu+\sigma_{i j}\right) Q_{i j} \\
& =b_{j} f_{i} q_{j i}-\left(2 \mu+\sigma_{i j}\right) Q_{i j}
\end{aligned}
$$

where $\Lambda_{i}, p_{i j}, q_{i j}$ may be complicated nonlinear functions of time and/or the state variables, and where we have made use of the conservation property (A3). 


\section{Recruitment}

The recruitment function is the key to the dynamics of extinction versus persistence, since we assume mortality is uniform across groups. If a group's recruitment does not keep up with the uniform mortality as well as other groups, it will not persist, if by persistence we mean proportional representation being bounded away from zero. If persistence is defined instead as population being bounded away from zero, even though proportionate population may go to zero, then persistence requires that recruitment outpace mortality solely within a group, not necessarily when compared with other groups.

As examples of the types of recruitment functions that might be easily incorporated into the model, consider

(a) maternal determination:

$$
\Lambda_{i}=\sum_{l} \sum_{k} r_{l k} Q_{l k} \delta(k, i)
$$

where $\delta(i, j)$ is 1 if $i=j, 0$ otherwise,

(b) biparental determination:

$$
\Lambda_{i}=\sum_{l} \sum_{k} r_{l k} Q_{l k}(\gamma \delta(k, i)+(1-\gamma) \delta(l, i))
$$

where $0 \leq \gamma \leq 1$,

(c) biparental determination influenced by outside population:

$$
\Lambda_{i}=\sum_{l} \sum_{k} r_{l k} Q_{l k}\left[\beta(\gamma \delta(k, i)+(1-\gamma) \delta(l, i))+(1-\beta) \frac{m_{i}+f_{i}+M_{i}+F_{i}}{\sum_{i} m_{i}+f_{i}+M_{i}+F_{i}}\right]
$$

where $0 \leq \beta \leq 1$, etc. More elaborate and germane functions may be conjured, based on what is known or conjectured about a particular system. In most cases, the values of the parameters are not generally known, but sometimes they are indeed quantifiable [4].

\section{Example: maternal determination}

As an example, consider the following model. Let the mortality and separation rates be the same for all groups, and let the type of the offspring be determined solely by the type of its mother, i.e.

$$
X_{j}^{l k}=\delta(k, j)
$$

where $\delta(i, j)$ is 1 if $i=j, 0$ otherwise. Furthermore, let the recruitment rate be directly proportional to the number of paired females of each type, with birth rates $r_{i}$. Assume some mixing functions $p_{i j}, q_{i j}$. Then the general model reduces to

$$
\dot{m}_{i}=r_{i} F_{i}-\left(\mu+c_{i}\right) m_{i}+(\mu+\sigma) M_{i}
$$




$$
\begin{aligned}
\dot{f}_{i} & =r_{i} F_{i}-\left(\mu+b_{i}\right) f_{i}+(\mu+\sigma) F_{i} \\
\dot{Q}_{i j} & =c_{i} m_{i} p_{i j}-(2 \mu+\sigma) Q_{i j} \\
& =b_{j} f_{i} q_{j i}-(2 \mu+\sigma) Q_{i j}
\end{aligned}
$$

Summing the $Q$ equations over $j$ and $i$ respectively, we obtain the aggregated model

$$
\begin{aligned}
\dot{m}_{i} & =r_{i} F_{i}-\left(\mu+c_{i}\right) m_{i}+(\mu+\sigma) M_{i} \\
\dot{f}_{i} & =r_{i} F_{i}-\left(\mu+b_{i}\right) f_{i}+(\mu+\sigma) F_{i} \\
\dot{M}_{i} & =c_{i} m_{i}-(2 \mu+\sigma) M_{i} \\
\dot{F}_{i} & =b_{i} f_{i}-(2 \mu+\sigma) F_{i}
\end{aligned}
$$

since, by definition, $\sum_{j} p_{i j}=\sum_{j} q_{i j}=1$. The aggregated model is linear, so its only equilibrium is zero, and all populations remain in a fixed ratio, so no populations go extinct unless all do. We can write (5) in matrix form,

$$
\dot{X}=A X
$$

where $X=\left(m_{1}, \ldots, m_{N}, f_{1}, \ldots, f_{N}, M_{1}, \ldots, M_{N}, F_{1}, \ldots, F_{N}\right)^{T}$, and where $A$, a $4 \mathrm{~N} \times 4 \mathrm{~N}$ matrix, can be written in block form as

$$
A=\left(\begin{array}{cccc}
B & 0 & C & D \\
0 & E & 0 & F \\
G & 0 & H & 0 \\
0 & J & 0 & K
\end{array}\right)
$$

where

$$
\begin{aligned}
B & =\operatorname{diag}\left(-\mu-c_{i}\right) \\
C & =\operatorname{diag}(\mu+\sigma) \\
D & =\operatorname{diag}\left(r_{i}\right) \\
E & =\operatorname{diag}\left(-\mu-b_{i}\right) \\
F & =\operatorname{diag}\left(\mu+\sigma+r_{i}\right) \\
G & =\operatorname{diag}\left(c_{i}\right) \\
H & =\operatorname{diag}(-2 \mu-\sigma) \\
J & =\operatorname{diag}\left(b_{i}\right) \\
K & =\operatorname{diag}(-2 \mu-\sigma)
\end{aligned}
$$

$A$ is easily triangulable by row operations:

$$
\tilde{A}=\left(\begin{array}{cccc}
B & 0 & C & D \\
0 & E & 0 & F \\
0 & 0 & H-\left[\frac{G}{B}\right] C & {\left[\frac{G}{B}\right] D} \\
0 & 0 & 0 & K-\left[\frac{J}{E}\right] F
\end{array}\right)
$$

where $[a / b]_{i j}=a_{i j} / b_{i j}$ if $i=j, 0$ otherwise, i.e. scalar division of nonzero matrix elements. The eigenvalues of $A$ must be the same as those of $\tilde{A}$, which are 
immediately seen to be

$\lambda=-\mu-c_{i}<0,-\mu-b_{i}<0, \frac{-\mu\left(2 \mu+\sigma+c_{i}\right)}{\mu+c_{i}}<0, \frac{b_{i}}{\mu+b_{i}}\left(\mu+\sigma+r_{i}\right)-(2 \mu+\sigma)$

The last $N$ eigenvalues may take on positive values if $r_{i}$ is sufficiently large. Thus the stability of the zero equilibrium will depend on the birth rates $r_{i}$.

A simple estimate for the spectrum of $A$ is given by observing that the first $3 N$ columns of $A$ are strictly diagonally dominant, and the last $N$ columns are strictly diagonally dominant if $\mu+\sigma+2 r_{i}<2 \mu+\sigma \quad \forall i$. That is, a necessary and sufficient condition for the strict diagonal dominance of $A$ is that $\mu>2 \max \left(r_{i}\right)$. Observing further that all the diagonal elements of $A$ are negative, and applying the Levy-Desplanques Theorem [7], we see that a sufficient condition for the zero solution to be stable is that $\mu>2 \max \left(r_{i}\right)$. This is simply a condition of mortality outpacing reproduction, and does not depend on pair formation rates at all. ¿From this analysis, we see that in systems where the recruitment rates can be written solely in terms of the aggregates $M_{i}, F_{i}, i=1, \ldots, N$, i.e. in systems where we need not keep track of different types of pairs, we need not keep track of pair formation functions $p_{i j}, q_{i j}$. This will not, however, generally be the case, since generally one's parentage will affect one's social traits. Analysis of most social transmission models will be far more complicated than this analysis.

\section{Discussion}

Previous approaches to modeling cultural transmission have either been very specific, as in the bilingual competition model of Baggs and Freedman [1], or have been general, but confined by a rigid framework. For example, Cavalli-Sforza and Feldman [4] and Boyd and Richerson [2] both construct an elaborate framework based on cultural transmission once per generation, e.g. at birth. This has the disadvantages of excluding cultural transmission during an individual's lifetime (e.g. religious conversion) and of requiring that generations be of equal duration, and generated simultaneously. The discrete-generation approach is not necessarily any easier to analyze than our differential equation approach, and it may introduce behaviors (e.g. periodicity) more appropriate to organisms which do have discrete, simultaneous generations, such as insects, than to humans.

The problems of cultural transmission are complex, and the data is minimal. We plan to continue this work in several directions, generally incorporating more realistic nonlinearities, tied to data, and analyzing the resulting more complicated systems with more numerically-oriented techniques, since it takes only one more complicated term in our equations to render their explicit analysis intractable. Most realistic models of cultural transmission will go beyond the proportionate mixing of our example of Section 4, and incorporate like-with-like preferences, i.e. $\phi_{i j} \neq 0$ in the pair formation terms. 
For example, a study by the Council of Jewish Federations [12] reports that the religion of the spouses of American Jewish subjects was Jewish in $89 \%$ of marriages before 1965 , and in $43 \%$ of marriages after 1985, although the proportion of Jews in the US population was only $2-3 \%$ during that period. This indicates a very large $\phi_{11}$ (preference of minority members for minority members) of 29 and 22 respectively, in comparison to the relatively indifferent $\phi_{00}$ (preference of majority members for majority members) of 1.0, during that period. Thus any serious modeling attempt must include like-with-like preferential mixing, where it is appropriate.

It will also be of interest to study various recruitment functions, as discussed in Section 3. It may also be fruitful to incorporate age structure into the framework, since cultural transmission often has an age-linked component [4].

\section{References}

[1] Baggs, I. and Freedman, H. I. (1990) A mathematical model for the dynamics of interactions between unilingual and a bilingual population: persistence vs. extinction. J. Math. Sociol. 16(1):51-75.

[2] Boyd, R. and Richerson P. J. (1985). Culture and the Evolutionary Process. The University of Chicago Press, Chicago.

[3] Castillo-Chavez, C. and Busenberg, S. (1991) On the solution of the two-sex problem. In: Proceedings of the Interantional Conference on Differential Equations and Applications to Biology and Population Dynamics (S. Busenberg and M. Martelli, eds.), Lecture Notes in Biomathematics, 80-98. Springer-Verlag, Berlin-HeidelbergNew York-London-Paris-Tokyo-Hong Konğ, Barcelona, Budapest.

[4] Cavalli-Sforza, L. L. and Feldman, M. W. (1981) Cultural Transmission and Evolution. Monographs in Population Biology 16. Princeton University Press, Princeton, New Jersey.

[5] Dietz, K. and Hadeler, K.P. (1988) Epidemiological models for sexually transmitted diseases. J. Math. Biol. 26, 1-25.

[6] Fredrickson, A.G. (1971) A mathematical theory of age structure in sexual populations: Random mating and monogamous marriage models. it Math. Biosci. 20, 117-143.

[7] R. Horn and C. Johnson. (1985) Matrix Analysis, Cambridge Univ. Press.

[8] Lotka A.J. Contributions to the analysis of malaria epidemiology. Am. J. Hygiene 3 (1923), Jan. suppl.

[9] Kendall, D.G. (1949) Stochastic processes and population growth. Roy. Statist. Soc., Ser. B 2, 230-264.

[10] McFarland, D. (1972) Comparison of alternative marriage models. In (Greville, T. N. E., ed.), Population Dynamics. Academic Press, New York London: 89-106.

[11] Parlett, B. (1972) Can there be a marriage function? In (Greville, T. N. E., ed.), Population Dynamics. Academic Press, New York London: 107-135.

[12] Steinfels, P. Debating intermarriage, and Jewish survival. New York Times Oct. 16, 1992 